\title{
Citizen science initiative points at childhood BCG vaccination as a risk factor for COVID-19
}

\author{
Jose de la Fuente ${ }^{1}$, Octavio Armas ${ }^{2}$, Luis Sanchez-Rodriguez ${ }^{2}$, Christian Gortazar ${ }^{1}$, and \\ Alexander Lukashev ${ }^{3}$
}

${ }^{1}$ IREC

${ }^{2} \mathrm{UCLM}$

${ }^{3}$ Sechenov University

December 31, 2020

\begin{abstract}
Current results do not provide conclusive evidence on the effect of BCG vaccination on COVID-19 alone or in combination with other factors. To address this limitation, in this study we used a citizen science initiative on the COVID-19 pandemic to collect data worldwide during October 2-30, 2020 (1,233 individuals) in a structured way for analyzing factors and characteristics of affected individuals in relation to BCG vaccination. For the first time, the results of our study suggested that vaccination with BCG may increase the risk for COVID-19 at certain age, particularly in individuals vaccinated at childhood. A reasonable explanation for this effect is the activation of certain innate immunity mechanisms associated with inflammatory reactions, which should be considered when analyzing the risks associated with this global pandemic. These factors should be considered when analyzing the risks associated with this global pandemic.
\end{abstract}

\section{Hosted file}

Rapid Communication.pdf available at https://authorea.com/users/332128/articles/501942citizen-science-initiative-points-at-childhood-bcg-vaccination-as-a-risk-factor-forcovid-19 


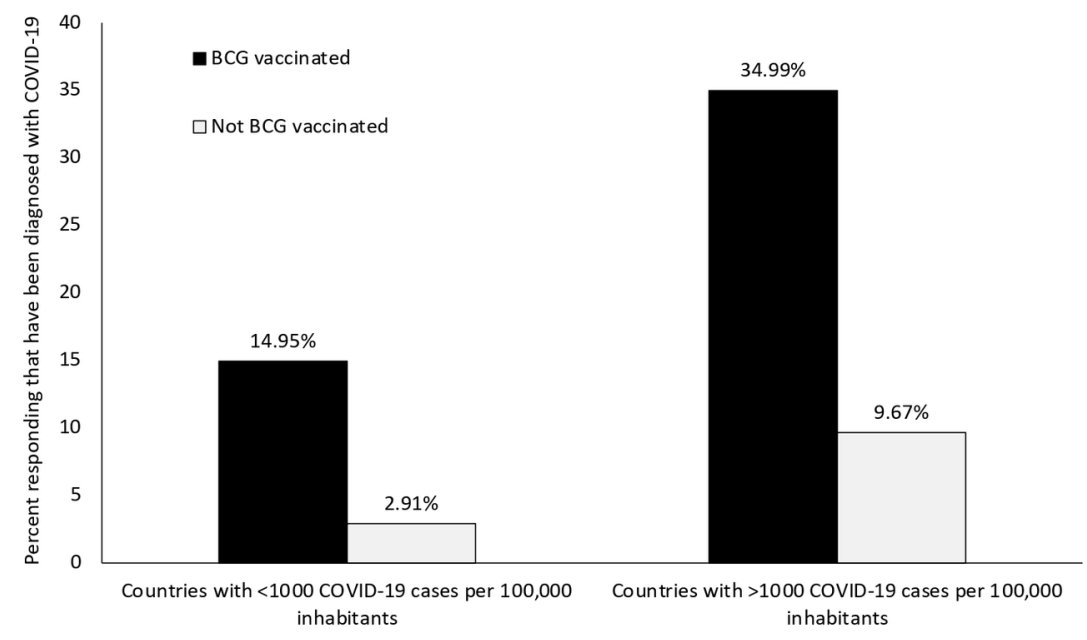

